\title{
Enhanced stability of layered phases in parallel hard spherocylinders due to addition of hard spheres
}

\author{
Zvonimir Dogic, ${ }^{1}$ Daan Frenkel, ${ }^{2}$ and Seth Fraden ${ }^{1}$ \\ ${ }^{1}$ The Complex Fluid Group, Martin Fisher School of Physics, Brandeis University, Waltham, Massachusetts 02254 \\ ${ }^{2}$ FOM-Institute for Atomic and Molecular Physics, Kruislaan 407, 1098 SJ Amsterdam, The Netherlands
}

(Received 31 March 2000)

\begin{abstract}
There is increasing evidence that entropy can induce microphase separation in binary fluid mixtures interacting through hard particle potentials. One such phase consists of alternating two-dimensional liquidlike layers of rods and spheres. We study the transition from a uniform miscible state to this ordered state using computer simulations, and compare results to experiments and theory. We conclude the following: (1) There is stable entropy driven microphase separation in mixtures of parallel rods and spheres. (2) Adding spheres smaller than the rod length decreases the total volume fraction needed for the formation of a layered phase, and therefore small spheres effectively stabilize the layered phase; the opposite is true for large spheres. (3) The degree of this stabilization increases with increasing rod length.

PACS number(s): 64.70.Md, 64.75. $+\mathrm{g}, 61.30 . \mathrm{Cz}$
\end{abstract}

\section{INTRODUCTION}

In hard particle fluids all allowed configurations have the same energy, and therefore it is the number of states, or equivalently the entropy of a system, that determines the equilibrium phase. Examples of well known phase transitions where the formation of ordered structures are driven solely by an increase in entropy are the liquid to crystal transition in hard spheres [1], and the isotropic to nematic [2] and the nematic to smectic transition in hard rods $[3,4]$. Because of their high degree of monodispersity, and because of the dominant role of steric repulsion in the pair potential, colloidal suspensions of polystyrene latex and rodlike viruses have often been used as experimental model systems for the study of entropy induced ordering in hard-sphere [5-7] and hard-rod systems, respectively $[8,9]$.

A natural extension of the above work is to the phase behavior of mixtures, with a number of recent experimental and theoretical studies focusing on the phase behavior of binary mixtures of hard spheres [10-19]. We have recently begun work on less studied systems that closely approximate hard-rod-hard-sphere and hard-rod-polymer mixtures [20$26]$. As a model for hard rods we used either the $f d$ or tobacco mosaic virus (TMV) virus; as a model for hard spheres we used polystyrene latex; and as a model for polymers we used poly(ethylene-oxide) with varying molecular weights $[27,28]$. The part of the phase diagram explored consisted of pure rods in either the isotropic, nematic, or smectic phase, to which a small volume fraction of spheres or polymers was added. Remarkably, besides the expected uniform mixtures and bulk demixing, we also observed a variety of microphases for a wide range of sphere sizes and concentrations [27]. In microphase separation the system starts separating into liquidlike regions that are rich in either spheres or rods. However, unlike bulk demixing, where rod and sphere rich regions grow until reaching macroscopic dimensions, in microphase demixing these liquidlike regions increase only to a critical size after which they order into well defined three-dimensional equilibrium structures. One of the microseparated phases observed, named the lamellar microphase, consisted of stacks of a two-dimensional liquidlike layer of parallel rods alternating with a layer of spheres. The subject of this paper is a theoretical analysis of phase transitions between the lamellar phase and either a phase consisting of a uniform mixture of spheres and rods in a nematic phase, named the miscible phase, or a phase where rods and spheres bulk phase separate into a rod-rich phase and sphererich phase named the immiscible phase as illustrated in Fig. 1.

In this paper we use the second virial approximation first studied by Koda et al. [29] to examine the influence of molecular parameters such as shape and size, on the phase behavior of rod-sphere mixtures. As the second-virial theory is approximate in nature, we validate the theoretical predictions by comparing them with either computer simulations or experimental results. The remainder of this paper is organized as follows: In Sec. II we formulate the second virial approximation for the rod-sphere mixture. The general features of the phase diagram are discussed, and a physical picture of the factors responsible for the enhanced stability of the layered phase due to the presence of spheres is presented. In Sec. III the influence of varying the spherocylinder length on the phase behavior of spherocylinder-sphere mixtures is studied using computer simulations, and the results are compared to theoretical predictions. Section IV examines how changes of the sphere diameter influence the phase behavior of spherocylinder-sphere mixtures. Finally in Sec. V we present our conclusions.

\section{GENERAL FEATURES OF A PHASE DIAGRAM OF A SPHEROCYLINDER-SPHERE MIXTURE}

Although the equilibrium phases of all hard particle fluids are determined by maximizing the entropy, ordering transitions are still possible because the expression for the total entropy, or equivalently free energy, splits into two parts. The ideal contribution to the entropy is of the form $\rho \ln \rho$, where $\rho$ is the density distribution function. This contribution to the entropy attains a maximum for a uniform density distribution, and therefore always suppresses transitions from uniform to modulated phases. In contrast, excluded volume entropy sometimes increases with increasing spatial organization, and therefore drives the system toward a modulated 

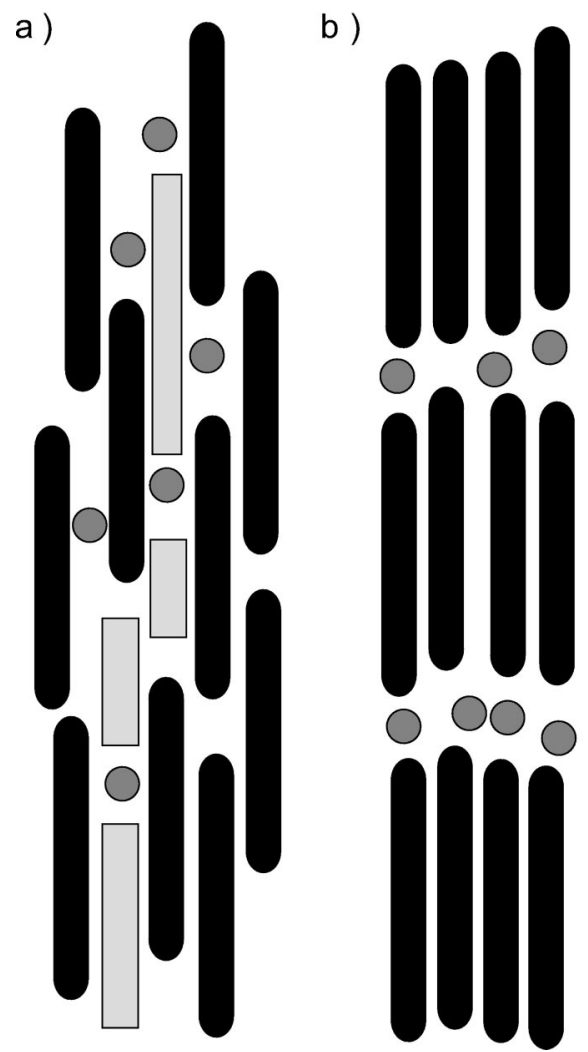

c)

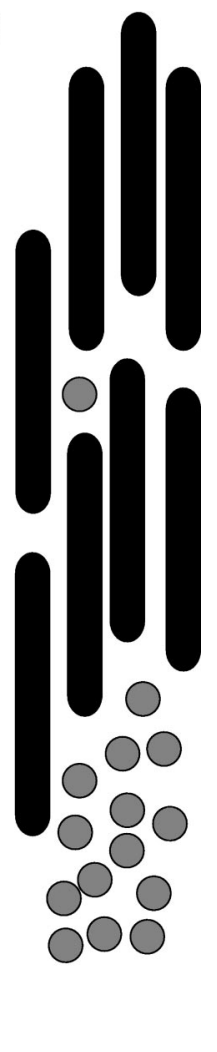

FIG. 1. (a) A schematic illustration of excluded volume effects in a miscible (nematic) phase in a spherocylinder-sphere mixture. In the miscible phase each sphere creates a large excluded volume around it, indicated by gray areas, that is inaccessible to spherocylinders. (b) When the system undergoes a transition to a lamellar (layered) phase, the large excluded sphere-spherocylinder volume vanishes since the probability distribution severely limits the number of ways that spheres are allowed to approach spherocylinders. (c) Illustration of the immiscible phase where the system bulk phase separates into a rod-rich phase and a sphere-rich phase.

phase. In this paper we use the highly simplified second virial approximation to calculate the excluded volume entropy.

The equilibrium phase in a spherocylinder-sphere mixture is determined by four parameters: the length over diameter of a spherocylinder $\left(L / D_{s c}\right)$, the diameter of a spherocylinder over the diameter of a sphere $\left(D_{s c} / D_{s p}\right)$, the total volume fraction of spheres and spherocylinders $(\eta)$, and the partial volume fraction of spheres $\left(\rho_{s p}\right)$. To help us in the interpretation of our results we first define the slope

$$
\tau=\lim _{\rho_{s p} \rightarrow 0} \frac{\eta\left(\rho_{s p}\right)-\eta(0)}{\rho_{s p}},
$$

where $\eta\left(\rho_{s p}\right)$ is the total volume fraction of the rod-sphere mixture at the layering transition after the introduction of spheres at the partial volume fraction $\rho_{s p}$ (see Fig. 2). A positive value of $\tau$ implies that adding a second component stabilizes the nematic phase by displacing the smectic transition to higher densities. For the case when both components are spherocylinders of different lengths but with the same diameter, slope $\tau$ is positive if the ratio of lengths is less than approximately $7[30,31]$. In the same manner, negative values of $\tau$ imply that the second component stabilizes

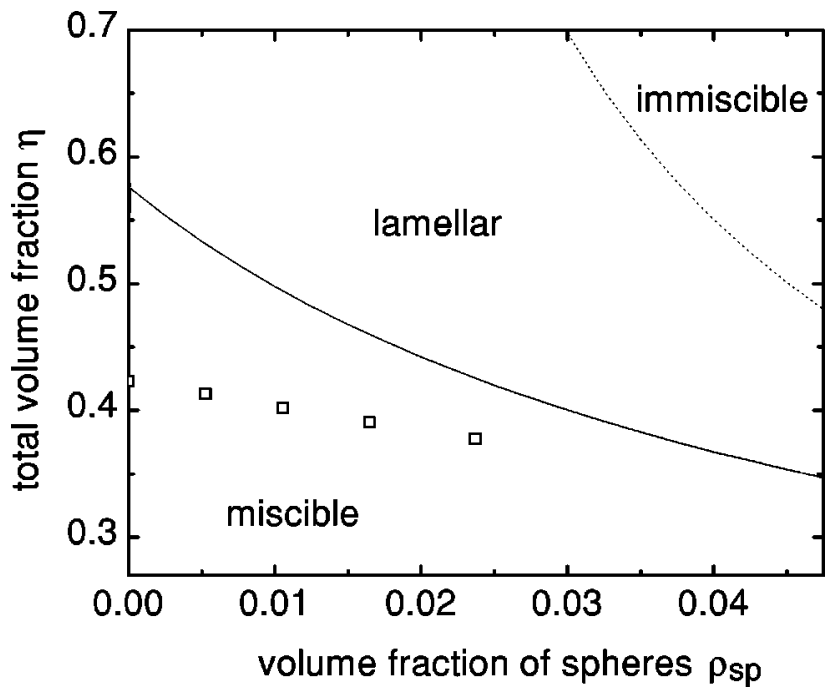

FIG. 2. Stability boundaries for a mixture of perfectly aligned spherocylinders $\left(L / D_{s c}=20\right)$ and spheres with equal diameter $\left(D_{s c} / D_{s p}=1\right)$. The full line indicates the theoretical prediction of the volume fraction at which the system becomes unstable toward lamellar fluctuations, and its slope is $\tau$ [Eq. (1)]. The dashed line indicates instability toward demixing into two macroscopically distinct phases. Squares are results of computer simulations at which the layering transition is observed. Theoretically, the periodicity associated with a one-dimensional lamellar instability continuously grows and diverges as the system completely phase separates. Illustrations of the miscible, immiscible, and lamellar phases are shown in Fig. 1.

the smectic phase. There are predictions of a negative value of $\tau$ in a bidisperse rod mixture when the ratio of rod lengths is large enough [30], or when added rods have a larger diameter [32]. In this section we focus on the phase behavior of the spherocylinder-sphere mixture for the specific microscopic parameters $L / D_{s c}=20$ and $D_{s c} / D_{s p}=1$. We present a physical picture of excluded volume effects that are responsible for the enhanced stability of the lamellar phase. In the next two sections we extend our study on how changes in the molecular parameters $L / D_{s c}$ and $D_{s c} / D_{s p}$ modify the phase behavior and, in particular, their influence on the magnitude and sign of the slope $\tau$.

\section{A. Second virial approximation}

The second virial approximation for a mixture of perfectly aligned spherocylinders and spheres of equal diameter was proposed by Koda, Numajiri, and Ikeda [29], and is generalized for arbitrary $L / D_{s c}$ and $D_{s c} / D_{s p}$ in the Appendix. It was previously shown that the second virial approximation qualitatively described the formation and various features of the smectic phase of hard rods [3,33-35]. Here we study how the addition of spheres perturbs the formation of the smectic phase. Since the sphere volume fraction is very low, we expect that the second virial approximation is still qualitatively correct for these mixtures. We consider a sinusoidal perturbation from the uniform density for both spherocylinders and spheres. From Eqs. (A3) and (A6) in the Appendix, we obtain the free energy difference between the uniformly mixed and layered state in a spherocylinder-sphere mixture: 


$$
\delta F=a_{1}^{2}\left(S_{11}-2 \frac{a_{1}}{a_{2}} S_{12}+\left(\frac{a_{1}}{a_{2}}\right)^{2} S_{22}\right)=0
$$

The phase diagram obtained within this approximation for microscopic parameters $L / D_{s c}=20$ and $D_{s c} / D_{s p}=1$ is shown in Fig. 2. From the phase diagram we see that the first prediction of the model is that spheres, upon addition to a smectic phase, will preferentially occupy space between smectic layers, and therefore create a stable microseparated lamellar phase. The second prediction is that the total volume fraction at which the system undergoes a transition from a uniform miscible state to a layered lamellar state is lowered by increasing the partial volume fraction of spheres. This implies that the slope $\tau$ is negative for this particular spherocylinder-sphere mixture, and we conclude that in this case spheres enhance the layering transition.

We can assign a simple physical origin to every term given in Eq. (2) above and Eq. (A5) of the Appendix. The parts of the spherocylinder-spherocylinder interaction term $S_{22}$ and sphere-sphere term $S_{11}$ that scale as $\eta$ are due to the ideal $(i d)$ contribution to the free energy, also known as the entropy of mixing and are denoted as $S_{22}^{i d}$ and $S_{11}^{i d}$, respectively. The terms having a $\eta^{2}$ dependence in $S_{22}, S_{12}$, and $S_{11}$ are due to the spherocylinder-spherocylinder, spherocylinder-sphere, and sphere-sphere excluded volume (ex) interactions, respectively, and are denoted as $S_{22}^{e x}, S_{12}^{e x}$, and $S_{11}^{e x}$. Since the instability is defined as $\delta F\left(\eta_{c}, k_{c}\right)=0$, at a critical density $\eta_{c}$ and at a critical wave vector $k_{c}$ all individual contributions to the free energy difference in Eq. (2) must add up to zero. In Fig. 3 we show the value of all terms with distinct physical origins at the instability density $\eta_{c}$ and wave vector $k_{c}$ as a function of partial volume fraction of spheres. Since from our analysis we cannot determine the absolute amplitude of $a_{1}$, we only plot the ratios of all free energy components to the absolute value of the spherocylinder-spherocylinder excluded volume $\left|S_{22}^{e x}\right|$. If we set the partial volume fraction of spheres to zero $\left(\rho_{s p}=0\right)$ in Eq. (2) we obtain an equation whose solution indicates the nematic-smectic stability limit in a pure suspension of aligned spherocylinders [33]. For these conditions the only two nonzero components of free energy are $S_{22}^{e x}$, which is negative and therefore drives the transition, and $S_{22}^{i d}$, which is positive and therefore suppresses the transition. As we start increasing the partial sphere volume fraction $\rho_{s p}$, the spherocylinder-sphere free volume term $S_{12}^{e x}$ rapidly assumes large negative values, as evidenced by the rapidly decreasing ratio of $S_{12}^{e x} /\left|S_{22}^{i d}\right|$. This implies that layering the mixture significantly decreases the excluded volume that is due to the spherocylinder-sphere interaction.

We can use the information gained from the second virial approximation to obtain a clear physical picture of excluded volume effects in spherocylinder-sphere mixtures, and explain the enhanced stability of the lamellar phase. Taking any single spherocylinder in a uniform spherocylindersphere mixture and replacing it by two spheres will leave the value of the excluded volume virtually unchanged. The reason for this lies in the fact that the volume excluded to the spherocylinder due to the presence of a sphere with equal diameter, under the constraint of uniform packing, is a spherocylinder with diameter $2 D_{s c}$ and length $\left(L+2 D_{s c}\right)$,

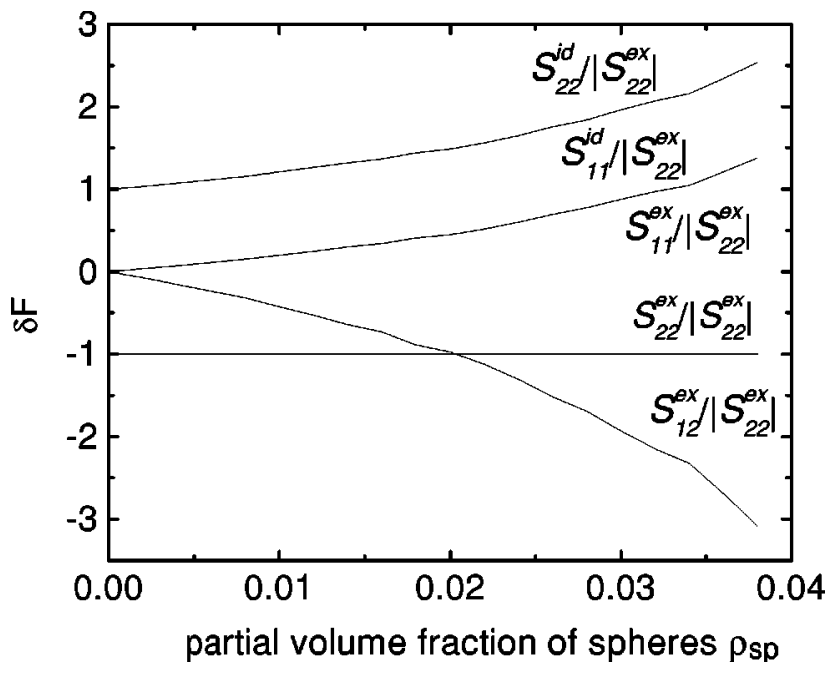

FIG. 3. Term-by-term dependence of the free energy difference between the miscible and lamellar phases [Eq. (2)] as a function of the partial volume fraction of spheres for $L / D_{s c}=20$ and $D_{s c} / D_{s p}$ $=1$. The $S_{11}^{i d}$ and $S_{22}^{i d}$ terms are the sphere and spherocylinder ideal contributions to the total free energy difference between the layered and uniform states. $S_{11}^{e x}, S_{12}^{e x}$, and $S_{22}^{e x}$ are excluded volume contributions to the free energy due to sphere-sphere, spherocylindersphere, and spherocylinder-spherocylinder interactions, respectively. Since from our analysis we cannot determine the amplitude, in Eq. (2) we plot amplitude independent ratios of each of five components of the free energy with different origins to the spherocylinder-spherocylinder excluded volume interactions. The stability condition is that $\delta F=0$, so for any value of partial volume fraction of spheres $\rho_{s p}$ the sum of the five contributions to $\delta F$ is zero. $\delta F$ of the ideal terms are positive; hence they stabilize the uniform, miscible nematic state, while the free volume terms are negative, favoring the lamellar state. The excluded volume spheresphere term $\left(S_{11}^{e x}\right)$ is negligible, and the spherocylinder-sphere $\left(S_{12}^{e x}\right)$ term dominates the transition.

where $L$ and $D_{s c}$ are defined in Fig. 4. However, the excluded volume between any two spherocylinders with large $L / D_{s c}$ is only about twice this value, as illustrated in Fig. 4. Although replacing spherocylinders by spheres in such a manner leaves the excluded volume almost unchanged, it significantly decreases the total volume fraction of the mix-

a)

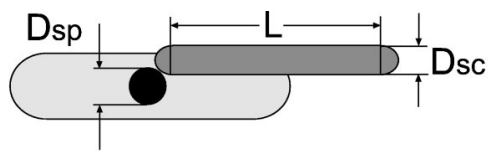

b)

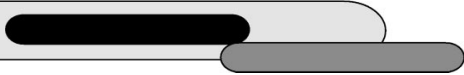

FIG. 4. (a) Volume excluded to the center of mass of a spherocylinder (sc) due to the presence of a sphere ( $\mathrm{sp}$ ) is indicated by light shading. (b) Volume excluded to the center of the mass of a second spherocylinder due to the presence of the first. Replacing a spherocylinder by a sphere decreases the excluded volume by approximately a factor of 2 , but it decreases the total volume fraction much more since the volume of a spherocylinder with large $L / D_{s c}$ is greater than the volume of a sphere with diameter $D_{s c}$. The comparatively large excluded volume between a sphere and a spherocylinder is the reason for the enhanced formation of the lamellar phase. 
ture since the volume of two spheres is much smaller than the volume of a spherocylinder with large $L / D_{s c}$. Therefore in the spherocylinder-sphere mixture we encounter excluded volume problems similar to those found in a pure spherocylinder solution, but at a lower total volume fraction. As in pure spherocylinders, the system reduces the excluded volume by undergoing a transition to a layered phase. The excluded volume is reduced in the lamellar state because a periodic density distribution forces spheres and spherocylinders into alternate layers thus decreasing the probability of the very unfavorable sphere-spherocylinder contacts, as illustrated in Fig. 1. This explains the large decrease in the value of the $S_{12}^{e x}$ term at the lamellar transition that we observe in the second virial theory. This term is responsible for the enhanced stability of the lamellar phase in a spherespherocylinder mixture. In conclusion, it is the inability to efficiently pack a uniform mixture of spherocylinders and spheres, as reflected in the large spherocylinder-sphere excluded volume term, that destabilizes the nematic phase and enhances the formation of a layered phase.

An alternate way to think about the formation of a layered phase is to focus on the effects of spherocylinder ends [36]. The nematic phase in our simplified model is characterized by random distribution of spherocylinders along their axial and radial directions, as illustrated in Fig. 5. This end effect is responsible for the formation of the smectic phase, which is characterized by a periodic density distribution. In a similar fashion, introducing a sphere into the nematic phase will have the same effect on the surrounding spherocylinders as another spherocylinder end. Therefore adding spheres very effectively increases the density of spherocylinder ends, and decreases the total volume fraction. To resolve the difficulties in efficient packing due to these extra spherocylinder ends, the mixture layers at a lower total volume fraction.

\section{B. Monte Carlo simulation}

In Sec. II A we discussed two predictions of the second virial theory for a spherocylinder-sphere mixture with $L / D_{s c}=20$ and $D_{s p} / D_{s c}=1$ : the existence of the lamellar phase and the enhanced stability of the lamellar phase when compared to a smectic phase of pure spherocylinders. Our results are in agreement with previous studies by Koda et al. [29]. However, the second virial approximation is highly approximate, and there is reasonable concern about the influence of higher terms on the topology of the phase diagram. To support their conclusions, Koda et al. performed computer simulations, which indicated the existence of a lamellar phase $[37,29]$. Still, the question of whether spheres simply fill the voids between layers in an already formed smectic phase, or actually induce layering at lower total volume fraction, was not addressed. In this section, using Monte Carlo simulations we address the question of the influence of adding spheres on the phase behavior of spherocylinders by determining the slope $\tau$ in Eq. (1) in a mixture of spherocylinders and spheres with parameters $L / D_{s c}=20$ and $D_{s p} / D_{s c}$ $=1$.

A Monte Carlo simulation of a mixture of hard-spheres and perfectly aligned hard spherocylinders was performed at a constant pressure and number of particles [38]. Most simulations contained 392 spherocylinders and a variable number

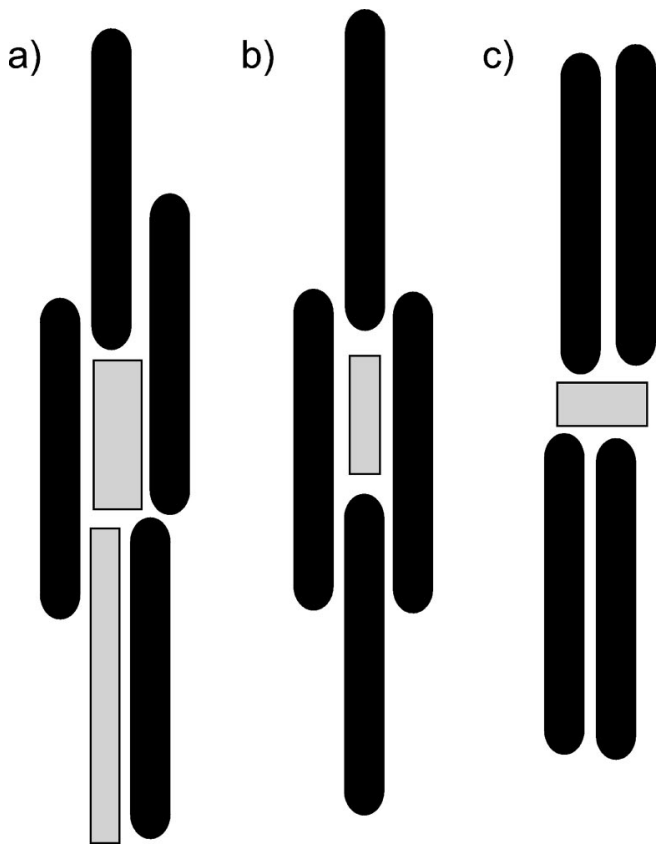

FIG. 5. (a) A schematic example of a typical configuration of spherocylinders in a dense nematic phase. Since the nematic phase is characterized by a uniform density distribution, this results in inefficient packing and large excluded volume between spherocylinders both along their radial and axial directions. This large and unfavorable excluded volume is indicated by lightly shadowed areas. (b) An illustration of a typical configuration of spherocylinders in a columnar phase where the excluded volume between spherocylinders is lower compared to the nematic phase at the same density, and the ideal part of free energy is higher. In a columnar phase the spherocylinders are forced into registry as one spherocylinder occupies space right above or below another one. Therefore, the columnar phase is characterized by two-dimensional order in the plane perpendicular to the spherocylinder's long axis, and onedimensional disorder parallel to the long axis. (c) A representative configuration of spherocylinders in a smectic phase, which is characterized by one-dimensional order along the spherocylinder's long axis and two-dimensional disorder in the perpendicular directions. Both theory and experiment indicate that the columnar phase is always metastable with respect to the smectic phase $[33,45]$.

of spheres. To check for finite size effects we also ran simulations with 784 spherocylinders, but saw no significant difference in the results obtained. In one sweep, pressure was increased from a dilute homogeneous mixture up to a well ordered, dense smectic or lamellar phase. At each value of the pressure, the density of spheres and spherocylinders and their corresponding smectic order parameter were measured after the system was allowed to equilibrate. Identical results were obtained when the pressure was slowly decreased from an initially dense phase composed of alternating layers of spherocylinders and spheres to a dilute homogeneous mixture.

Besides lamellar transitions, there is a possible demixing transition where spherocylinders and spheres phase separate into macroscopically distinct phases. However, once a layered phase is formed the exchange of spheres between layers drops to a negligible amount, leaving open the possibility that system would undergo a demixing transition, but is stuck in a lamellar phase, which is only a metastable state. 


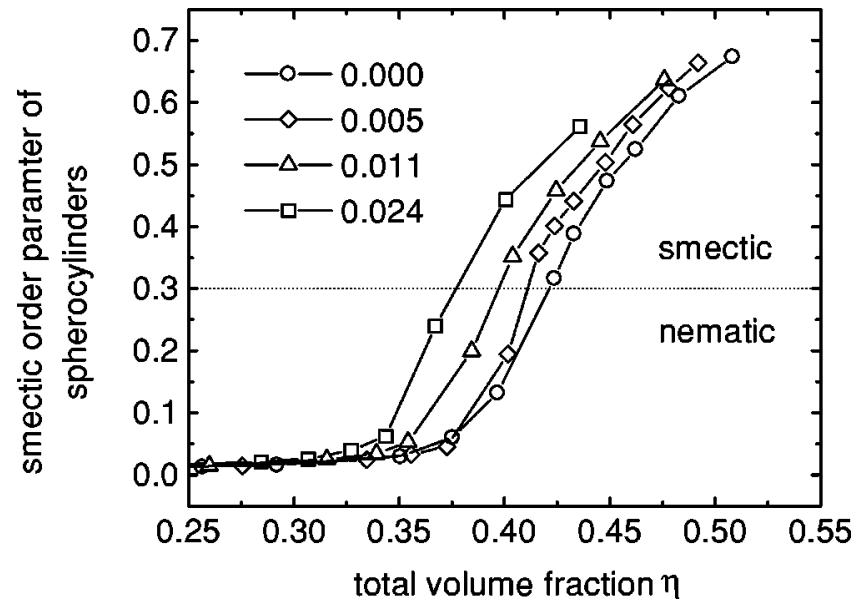

FIG. 6. Smectic order parameter obtained from Monte Carlo simulations is plotted against the total volume fraction for spherocylinders $(\eta)$ with $L / D_{s c}=20$. From right to left, the partial volume fraction of spheres $\left(\rho_{s p}\right)$ increases from $0 \%$ to $2.4 \%$, as indicated by the legend. The phase diagram is reconstructed from these data by defining a phase as layered when the spherocylinder order parameter reaches a value of 0.3 .

To find the location of the demixing transition it is necessary to measure the chemical potential of both spherocylinders and spheres in a spherocylinder-sphere mixture [39]. This possibility was not examined in this work, primarily because we are only interested in how low concentrations of spheres perturb the formation of the layered phase. Therefore, it is reasonable to expect that at a very low volume fraction of spheres, the lamellar transition is going to be more stable than the demixing transitions as predicted by the second virial theory.

A plot of the smectic order parameter for spherocylinders with $L / D_{s c}=20$ as a function of increasing total density for different partial volume fractions of spheres is shown in Fig. 6. As the system approaches a certain critical density, we observe a rapid nonlinear increase in the smectic order parameter, that we interpret as a signature of the nematic to smectic phase transition. This critical density shifts to lower values of the total volume fraction as the partial volume fraction of spheres is increased. To reconstruct a phase diagram from the above data, we define a phase as layered when its smectic order parameter reaches a value of 0.3 [40]. For a pure spherocylinder suspension this value yields good agreement with previous studies of the volume fraction of the nematic-smectic phase transition [41]. Since we are mostly interested in the qualitative behavior of a spherocylindersphere mixture, this method should suffice for our purposes. Using this phenomenological rule, the phase diagram for a mixture of spherocylinders and spheres $\left(L / D_{s c}\right.$ $\left.=20, D_{s c} / D_{s p}=1\right)$ is reconstructed and compared to the second virial theory in Fig. 2. An immediate conclusion drawn from Fig. 2 is that adding spheres to aligned spherocylinders enhances the stability of the lamellar phase, which is indicated by the negative value of slope $\tau$, in agreement with the prediction of the second virial approximation.

\section{EFFECTS OF SPHEROCYLINDER LENGTH ON THE PHASE DIAGRAM}

Next we proceed to investigate the influence of varying the spherocylinder length on the magnitude of slope $\tau$. The
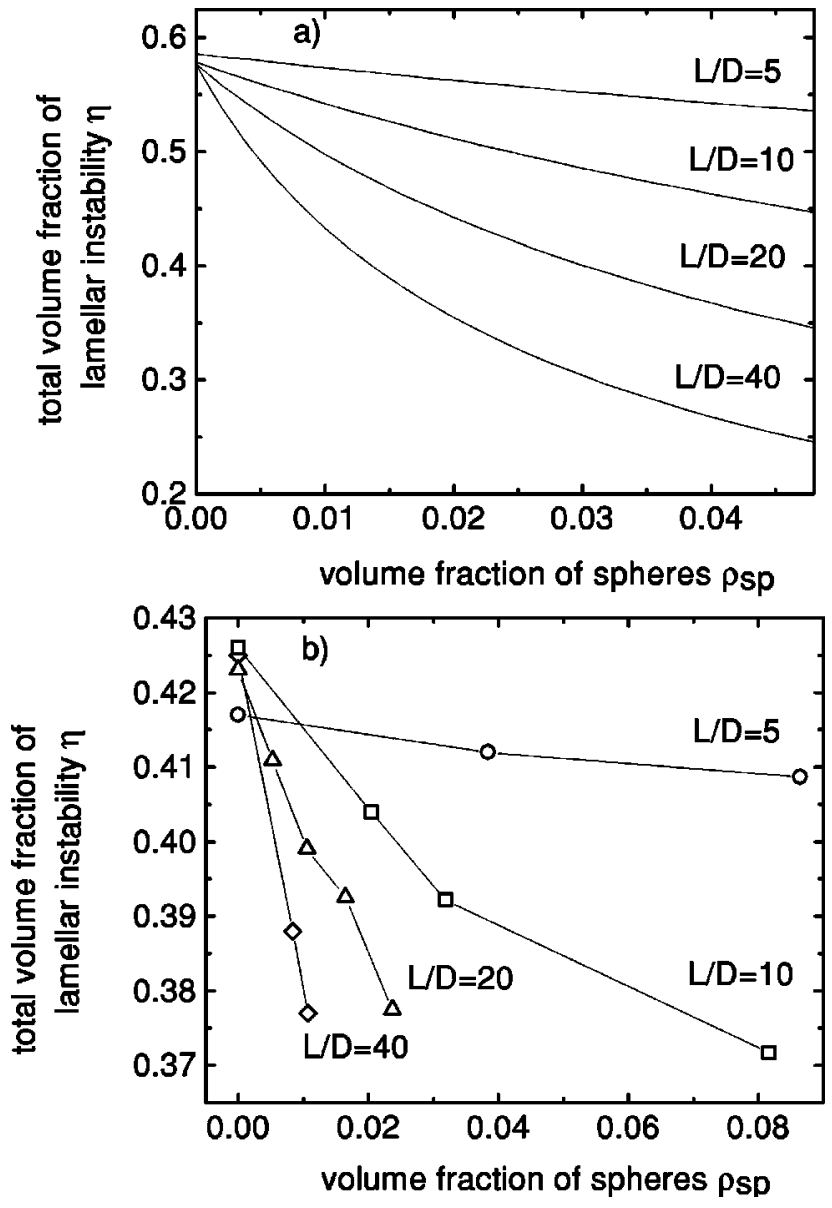

FIG. 7. (a) Prediction from the second virial theory for the total volume fraction $(\eta)$ of the lamellar instability as a function of sphere partial volume fraction $\left(\rho_{s p}\right)$ for spherocylinders with different $L / D_{s c}$ ratios. The diameter of spherocylinders is kept constant, and is equal to the diameter of the spheres. (b) Results from Monte Carlo simulations for the lamellar instability of spherocylinders as a function of partial volume fraction of spheres for same conditions as in (a). The volume fraction at the phase transition was defined as having a smectic order parameter of spherocylinders equal to 0.3

predictions of the second virial theory for the nematiclamellar instability are shown in Fig. 7(a). The second virial theory clearly predicts the increasing stability of the lamellar phase with the increasing length of spherocylinder. To verify this prediction we repeated Monte Carlo simulations for spherocylinders with different $L / D_{s c}$, and used the same rule as before to identify the volume fraction of the nematiclamellar transition. The simulation results for the location of the nematic to layered transition are shown in Fig. 7(b). We can conclude that our simulations confirm predictions of the second virial model, and that the length of the spherocylinder is an important parameter in forming the lamellar phase, with longer spherocylinders showing an increasing tendency to form a layered phase at a lower volume fraction of added spheres.

Using the physical picture of the excluded volume effects developed in Sec. II provides a natural explanation for our simulation results in Fig. 7. With increasing spherocylinder length the excluded volume due to the spherocylinder-sphere interaction grows proportionally to the spherocylinder 


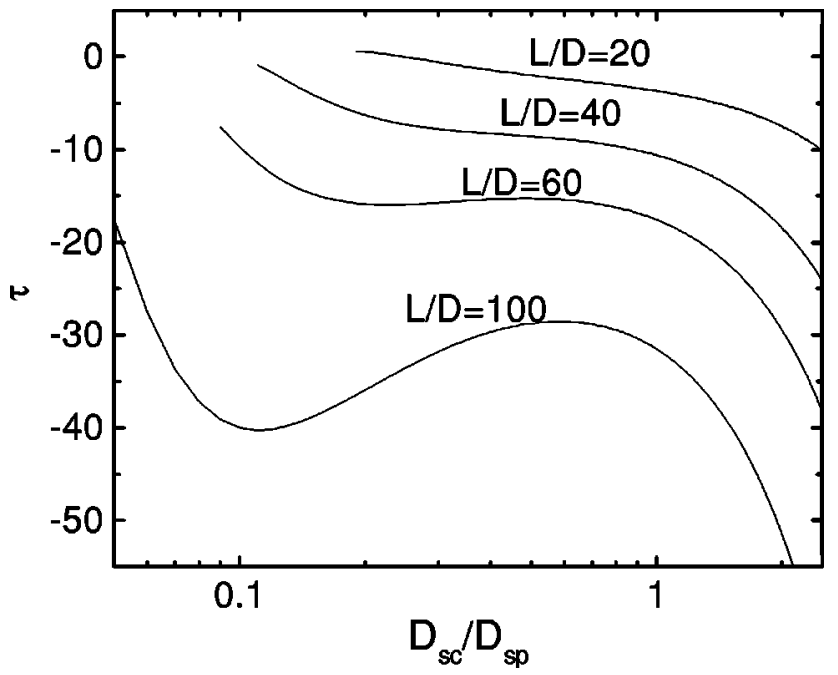

FIG. 8. Theoretical prediction for the stability criterium of the lamellar phase $\tau$ in Eq. (1) as a function of spherocylinder (sc) to sphere (sp) diameter ratio for four spherocylinders with different $L / D_{s c}$. The negative value of slope $\tau$ indicates that spheres of that particular size enhance the layering transition. Larger negative values of $\tau$ implies the formation of the lamellar phase at a lower total volume fraction.

length, and consequently the value of the $S_{12}^{e x}$ term increases in magnitude. As we have seen before, the larger the $S_{12}^{e x}$ term, the more likely it is for the system to form a layered phase.

It is interesting to consider the limit of spherocylinders with infinite aspect ratio. In the density regime of the nematic-smectic transition, this model can be mapped onto a system with skewed cylinders with an aspect ratio close to 1 . The nematic-smectic transition in this model was studied numerically $[42,43]$. If we consider the addition of spheres to this system, then the same affine transformation that maps the infinite spherocylinders onto squat, skewed spherocylinders, will map the spheres onto infinitely thin, parallel disks. As the disks are infinitely thin, they do not interact with each other but only with the cylinders. Inside the nematic phase, most volume is excluded for these disks. However, in the smectic phase, there is ample space for the disks between the layers. In fact, the stronger the layering, the larger the accessible volume. Hence, in this limit, the addition of spheres will strongly stabilize the smectic phase.

\section{EFFECTS OF SPHERE DIAMETER ON THE PHASE DIAGRAM}

In this section we investigate the influence of sphere diameter on the value of slope $\tau$. Figure 8 shows the prediction of the second virial theory for the dependence of slope $\tau$ on the ratio of spherocylinder to sphere diameter $\left(D_{s c} / D_{s p}\right)$ for spherocylinders with different $L / D_{s c}$. In Sec. IV A we examine the phase behavior of sphere-spherocylinder mixtures when the sphere diameter is smaller than spherocylinder diameter, and in Sec. IV B we examine the other case when the sphere diameter is larger than the spherocylinder diameter. In our model the presence of the spheres cannot alter the orientational distribution function of spherocylinders, which are always perfectly parallel to each other. It is reasonable to expect that this assumption holds for spheres smaller than the spherocylinder length, but as a sphere becomes larger than the spherocylinder length, long wavelength elastic effects start to dominate the behavior of the system, and hard spherocylinders will tend to align parallel to the surface of the sphere [44]. Therefore, in Fig. 8 we plot the values of slope $\tau$ only for those values of $D_{s c} / D_{s p}$ for which our assumptions are at least qualitatively correct. As we increase the sphere size beyond this limit, our model describes a highly artificial system of large spheres and parallel spherocylinders. In this regime we observe oscillations in the value of slope $\tau$ similar to what is observed in binary mixtures of parallel spherocylinders [30].

\section{A. Sphere diameter smaller than spherocylinder diameter}

In the regime where $D_{s c} / D_{s p}>1$ (for spherocylinders of any $L / D_{s c}$ ), decreasing the sphere size increases the stability of the lamellar phase as indicated by the increasing negative value of slope $\tau$ seen on the right hand side of Fig. 8. This prediction of the theory has a simple explanation in our picture of excluded volume in a sphere-spherocylinder mixture. If we halve the sphere radius $D_{s p}$, while keeping constant the volume fraction of spheres, we increase the number of spheres eight times. At the same time, the result of reducing the sphere size is to decrease the excluded volume of the spherocylinder-sphere interaction. However, the eightfold increase in the number of spherocylinder-sphere interactions more than compensates for the decrease in excluded volume between the sphere and spherocylinder, and consequently the magnitude of $S_{12}^{e x}$ increases with decreasing sphere diameter. This leads to the increased stability of the layered phase with decreasing sphere size.

It becomes difficult to verify this prediction using computer simulations. As the sphere size decreases at a constant total volume fraction $\eta$, the number of particles in a simulation rapidly reaches the order of thousands, requiring simulation times that are prohibitively long. As the ratio of spherocylinder to sphere diameter $\left(D_{s c} / D_{s p}\right)$ was varied within the accessible range between 0.5 to 2 , we did not observe any changes in the value of slope $\tau$ that were larger than our measurement error. Larger and longer simulations are needed for a careful analysis of spherocylinder-sphere mixtures with extreme values of the ratio $D_{s c} / D_{s p}$.

\section{B. Sphere diameter larger than spherocylinder diameter}

For spherocylinders with small $L / D_{s c}$, Fig. 8 shows that the magnitude of slope $\tau$ uniformly decreases with increasing sphere size. Eventually the slope $\tau$ changes sign and becomes positive, implying that large spheres stabilize the nematic phase and not the smectic phase. The phase diagram under conditions where slope $\tau$ is positive is shown in Fig. 9 . The wave vector associated with the layering transition, indicated by a solid line in Fig. 9, remains at an almost constant value. Another important point is that the amplitude ratio in Eq. (A6) is positive. This means that the periodic density modulations of the spherocylinders and spheres are in phase, which implies that spheres no longer go into the gap between two spherocylinder layers, but rather fit into the spherocylinder layer. However, as the partial volume fraction of spheres $\left(\rho_{s p}\right)$ is increased further, we observe a discon- 


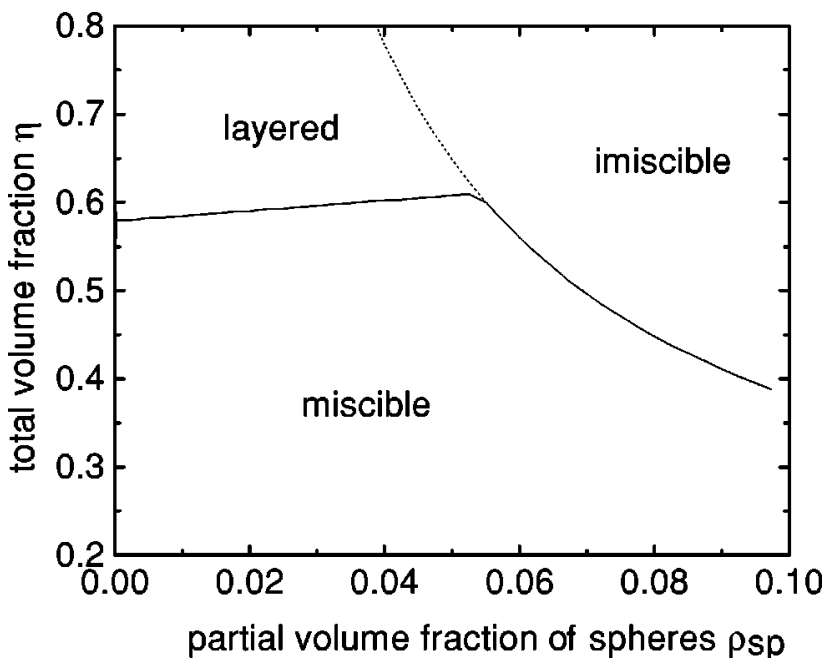

FIG. 9. Stability diagram of a mixture of spherocylinders $\left(L / D_{s c}=10\right)$ and large spheres $D_{s c} / D_{s p}=0.15$. Unlike a mixture of small spheres and spherocylinders (Fig. 2), introducing large spheres displaces the layering transition to higher total volume fractions, indicating a positive value of slope $\tau$. The structure of the layered phase is also different, with large spheres fitting in the smectic layer rather than into the smectic gap. The smectic periodicity associated with the layering transition does not change significantly until the concentration of spheres is high enough for the system to demix. Then the smectic wave vector discontinuously jumps to zero.

tinuous jump in the wave vector to a zero value. This implies that there is a discontinuous change from a layering to a demixing transition. As the demixing transition is reached there is also a change in sign of the amplitude ratio, which becomes negative, and the spherocylinders and spheres bulk separate. In contrast, the phase diagram for mixtures of small spheres and spherocylinders shown in Fig. 2 looks quite different. The amplitude ratio for this case is always negative, implying the formation of the lamellar phase. Another contrast is that in a mixture of small spheres and spherocylinders the wave vector associated with the layering transition decreases in a continuous fashion, until it reached zero value.

We now examine the behavior of individual terms in Eq. (2) for the mixture of large spheres and short spherocylinders shown in Fig. 9. Most notably, we find that at low volume fractions of spheres, where the system undergoes the layering transition, the ratio $S_{12}^{e x} / S_{22}^{e x} \ll 1$. This implies that upon layering there is almost no reduction of the unfavorable sphere-spherocylinder interaction, and that the spherocylinder-spherocylinder interaction alone drives the formation of the layered phase. In contrast, for small spheres this ratio was large, and was responsible for the enhanced stability of the lamellar phase, as shown in Fig. 3. At a higher volume fraction of large spheres where the mixture directly bulk phase separates, we find that the ratio $S_{12}^{e x} / S_{22}^{e x}$ $\gg 1$. This implies, as expected, that demixing very effectively reduces the unfavorable sphere-spherocylinder interactions. These results suggest a physical picture of the excluded volume effect. Unlike small spheres, large spheres cannot fit into the gap between smectic layers, and consequently there is no way to gain free volume by undergoing a layering transition. As an alternative, to gain free volume the system bulk phase separates at the lowest volume fraction of spheres possible.

While for short spherocylinders the magnitude of slope $\tau$ uniformly decreases with increasing sphere size, longer spherocylinders exhibit a qualitatively different behavior. For a mixture of spherocylinders with $L / D_{s c}=100$ and spheres with $D_{s c} / D_{s p}=0.1$, there is a pronounced increase in the stability of the lamellar phase, as shown in Fig. 8. By increasing the length of spherocylinders to even larger values, the region of increased stability of the lamellar phase shifts to higher values of the sphere radius. Two conditions emerge, which when satisfied lead to enhanced stability of the lamellar phase. First, it is necessary for a sphere to fit between two smectic layers without disturbing them. This condition is satisfied when $D_{s p} / L \approx 0.1$. The second condition is that $D_{s p} / D_{s c} \gg 1$. It was argued before that under these conditions large spheres are able to induce smectic correlations among neighboring spherocylinders [27], which in turn can enhance the formation of the lamellar phase.

Because of the large size asymmetry it was not feasible to carry out simulations for mixture of spherocylinders and spheres with $L / D_{s c} \approx 100$ and $D_{s c} / D_{s p} \approx 0.1$. However, these conditions are closely approximated by recent experiments on rodlike $f d\left(L=1 \mu \mathrm{m}, L / D_{s c} \approx 100\right)$ and polystyrene spheres [27]. Therefore, we compare theoretical results of slope $\tau$ for spherocylinders with $L / D_{s c}=100$, shown in Fig. 8, to these experimental results [27]. When large spheres $D_{s p} \approx 1 \mu \mathrm{m}\left(D_{s c} / D_{s p} \approx 0.01\right)$ are mixed with $f d$ at any concentration for which the nematic phase is stable, we observe no formation of the layered phase. Instead, large spheres phase separate into dense aggregates elongated along the nematic director, indicating that the value of slope $\tau$ is larger than zero. When the size of the sphere was decreased to $D_{s p}=0.1 \mu \mathrm{m}\left(D_{s c} / D_{s p} \approx 0.1\right)$, we observed a transition to a layered state at an $f d$ concentration of $20 \mathrm{mg} / \mathrm{ml}$. The formation of a smectic phase in a pure $f d$ suspension at the same ionic strength occurs at $65 \mathrm{mg} / \mathrm{ml}$. The fact that adding spheres diminishes the rod density by a factor of 3 indicates a large negative value of slope $\tau$. As the sphere size was further decreased $D_{s p}=0.022 \mu \mathrm{m}\left(D_{s c} / D_{s p}=0.46\right)$, there was again an indication of a lamellar phase, but this time at a much higher concentration of rods of about $50 \mathrm{mg} / \mathrm{ml}$. Thus, although small spheres still stabilize the layering transition, implying a negative value of slope $\tau$, the magnitude of slope $\tau$ is much less for $D_{s c} / D_{s p} \approx 0.46$ than for $D_{s c} / D_{s p}$ $\approx 0.1$. These qualitative trends of the nonmonotonic behavior of slope $\tau$ with sphere size observed in experiments of $f d$ polystyrene mixtures are very similar to the theoretical prediction shown in Fig. 8 for spherocylinders with $L / D_{s c}$ $=100$.

\section{CONCLUSIONS}

In this paper we have presented the predictions of the second virial theory for a mixture of parallel hard spherocylinders and hard spheres undergoing one-dimensional microphase separation. We have been able to verify a number of these predictions using Monte Carlo simulations. We found that spheres induce layering, which implies a negative value of the slope $\tau$, which is the change in total volume fraction of the mixture at the point of nematic-smectic insta- 
bility with respect to the partial volume fraction of added spheres [Eq. (1)]. At the same time the magnitude of the slope $\tau$ increases with increasing spherocylinder length. In other words, spheres at the same partial volume fraction stabilize layering of longer spherocylinders more than shorter spherocylinders. In addition, the theory predicts an unusual nonmonotonic behavior in slope $\tau$ as a function of sphere to spherocylinder diameter. Although the physical origin of this effect is not clear, it is intriguing that similar qualitative trends are observed in experiments of mixtures of the spherocylinderlike $f d$ and polystyrene spheres. However, in real experiments spherocylinders are free to rotate, are flexible, and have charge associated with them. Before quantitative comparisons with experiments are possible, it will be necessary to perform simulations and formulate theories that take into account these effects mostly ignored in this highly idealized treatment.

\section{ACKNOWLEDGMENTS}

We acknowledge useful discussions with Richard Sear and Bulbul Chakraborty. We thank Tomonori Koda for a critical reading of the manuscript. This research was supported by NSF Grant Nos. DMR-9705336 and NSF INT9113312. The work of the FOM Institute is supported by FOM ("Stichting Fundamenteel Onderzoek der Materie") with financial aid from NWO ("Nederlandse Organisatie voor Wetenschappelijk Onderzoek").

\section{APPENDIX}

A general expression for the free energy of bidisperse mixture at the second virial level is

$$
\begin{aligned}
\beta F\left(\rho_{1}, \rho_{2}\right)= & \sum_{i=1,2} \int_{V} d(\mathbf{r}) \rho_{i}(\mathbf{r}) \ln \left(\rho_{i}(\mathbf{r})\right) \\
& -\frac{1}{2} \sum_{\mathbf{i}=\mathbf{1}, \mathbf{2}} \sum_{\mathbf{j}=\mathbf{1}, \mathbf{2}} \int_{\mathbf{V}} d \mathbf{r}_{\mathbf{1}} \\
& \times \int_{V} d \mathbf{r}_{\mathbf{2}} \rho_{i}\left(\mathbf{r}_{\mathbf{1}}\right) \rho_{j}\left(\mathbf{r}_{\mathbf{2}}\right) \mathbf{f}_{\mathbf{i}, \mathbf{j}}\left(\mathbf{r}_{\mathbf{1}}, \mathbf{r}_{\mathbf{2}}\right),
\end{aligned}
$$

where the function $f_{i, j}$ is the overlap function between two spheres, the sphere and the spherocylinder, or two spherocyl- inders [29]. It attains the value of -1 if two particles overlap; otherwise it is equal to 0 . The terms involving $\rho \ln \rho$ represent the entropy of mixing, while the terms involving $f_{i, j}$ represent the free volume entropy. Since we are interested in one-dimensional layering, we look at the response of the system to the following density perturbations:

$$
\begin{aligned}
& \delta \rho_{1}(z)=a_{1} \cos \left(k_{z} z\right), \\
& \delta \rho_{2}(z)=a_{2} \cos \left(k_{z} z\right) .
\end{aligned}
$$

The free energy difference between the uniform and perturbed state is

$$
\delta F=F\left(1+\delta \rho_{1}(z), 1+\delta \rho_{2}(z)\right)-F(1,1)=\widetilde{\mathbf{a} S} \mathbf{a},
$$

where $\tilde{\mathbf{a}}=\left(a_{1}, a_{2}\right)$, and $\mathbf{S}$ is a two-dimensional stability matrix. To find the limit of stability we have to solve the equation $\operatorname{det}(\mathbf{S})=0$. For later convenience, we define the function

$$
\begin{aligned}
S\left(\frac{L}{D_{s c}}, \sigma, k\right)= & \frac{3 \sin \left[k \sigma\left(2+2 \frac{L}{D_{s c}}\right)\right]}{4 k^{3}} \\
& -\frac{2 k \sigma \cos \left[k \sigma\left(2+2 \frac{L}{D_{s c}}\right)\right]-\sin \left(k 2 \sigma \frac{L}{D_{s c}}\right)}{4 k^{3}} .
\end{aligned}
$$

The above expression depends only on geometrical factors, and is related to the Fourier transform of the spherocylinder which is specified by the excluded volume between a sphere of diameter $D_{s p}$ and a spherocylinder of length $L$ and diameter $D_{s c}$. Wave vector $k$ is dimensionless because it is rescaled with the spherocylinder diameter $\left(D_{s c}\right)$. The parameter $\sigma$ is defined as ratio of sphere diameter to spherocylinder diameter $\left(\sigma=D_{s p} / D_{s c}\right)$. In the limit of $L / D_{s c} \rightarrow 0$, the above expression reduces to a Fourier transform of a sphere with unit diameter. The stability matrix $\mathbf{S}$ for a mixture of spherocylinders and spheres has the form

$$
\mathbf{S}=\mid \begin{gathered}
\frac{\eta\left(1-\rho_{s p}\right)\left(1+4\left(1-\rho_{s p}\right) \eta S(0,1, k)\right)}{4} \\
\frac{2 \rho_{s p}\left(1-\rho_{s p}\right) \eta^{2} S\left(\frac{L}{D_{s c}}, 1+\sigma, k\right)}{\sigma^{6}\left(\frac{2}{3} \frac{L}{D_{s c}}+1\right)^{2}}
\end{gathered}
$$

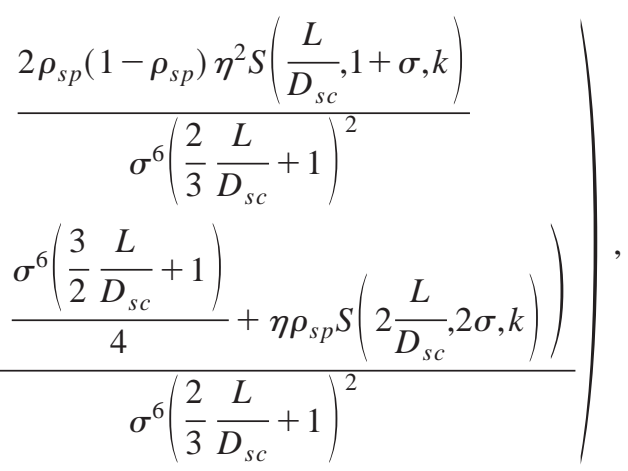


where $\rho_{s p}$ denotes the partial volume fraction of spheres, and varies between 0 and 1 , while $\eta$ denotes total volume fraction. Note that the terms in matrix elements $S_{11}$ and $S_{22}$, proportional to $\eta$, are due to configurational entropy, while terms proportional to $\eta^{2}$ are due to free volume entropy. As $k \rightarrow 0$ the condition $\operatorname{det}(\mathbf{S})=0$ reduces to the usual thermodynamic condition for the stability of the system against bulk phase separation.

To reconstruct the stability diagram from the determinant, we slowly increase the total volume fraction $\eta$. At a certain value of total volume fraction $\left(\eta_{c}\right)$, the determinant of $\mathbf{S}$ will equal zero for a specific wave vector $\left(k_{c}\right)$. If the wave vector $k_{c}$ obtained has a finite value, it implies that the system is undergoing a layering transition. On the other hand, the condition $\operatorname{det}(\mathbf{S})=0$, when $k_{c}=0$, implies a complete demixing. Once we obtain values of $\eta_{c}$ and $k_{c}$ we can find the ratio of amplitudes from the following formula:

$$
\frac{a_{1}}{a_{2}}=-\frac{S_{12}\left(\eta_{c}, k_{c}\right)}{S_{11}\left(\eta_{c}, k_{c}\right)} .
$$

A positive value of the amplitude ratio implies that the spheres and spherocylinders are in the same layer (the periodic modulations are in phase), while a negative value implies that the spheres and spherocylinders intercalate (the periodic modulations are out of phase).
[1] B.J. Alder and T.E. Wainwright, J. Chem. Phys. 27, 1207 (1957).

[2] L. Onsager, Ann. (N.Y.) Acad. Sci. 51, 627 (1949).

[3] M. Hosino, H. Nakano, and H. Kimura, J. Chem. Phys. 46, 1709 (1979).

[4] D. Frenkel, H. Lekkerkerker, and A. Stroobants, Nature (London) 332, 822 (1988).

[5] P.A. Forsyth, Jr., S. Marcelja, D.J. Mitchell, and B.W. Ninham, Adv. Colloid Interface Sci. 9, 37 (1978).

[6] R. Piazza, T. Bellini, and V. Degiorgio, Phys. Rev. Lett. 71, 4267 (1993).

[7] M.A. Rutgers et al., Phys. Rev. B 53, 5043 (1996).

[8] S. Fraden, in Observation, Prediction, and Simulation of Phase Transitions in Complex Fluids, edited by M. Baus, L.F. Rull, and J.P. Ryckaert (Kluwer, Dordrecht, 1995), pp. 113-164.

[9] R.B. Meyer, in Dynamics and Patterns in Complex Fluids, edited by A. Onuki and K. Kawasaki (Springer-Verlag, Berlin, 1990), p. 62.

[10] T. Biben and J.P. Hansen, Phys. Rev. Lett. 66, 2215 (1991).

[11] A.D. Dinsmore, A.G. Yodh, and D.J. Pine, Phys. Rev. E 52, 4045 (1995).

[12] A. Imhof and J.K.G. Dhont, Phys. Rev. Lett. 75, 1662 (1995).

[13] M.D. Eldrige, P.A. Madden, and D. Frenkel, Nature (London) 365, 35 (1993).

[14] S. Asakura and F. Oosawa, J. Polym. Sci. 33, 183 (1958).

[15] A.P. Gast, W.B. Russel, and C.K. Hall, J. Colloid Interface Sci. 109, 161 (1986).

[16] E.J. Meijer and D. Frenkel, J. Chem. Phys. 100, 6873 (1994).

[17] H.N.W. Lekkerkerker, in Structure and Dynamics of Strongly Interacting Colloids and Supramolecular Aggregates in Solution, edited by S.H. Chen, J.S. Huang, and P. Tartaglia (Kluwer, Dordrecht, 1992), p. 97.

[18] M. Dijkstra and R. van Roij, Phys. Rev. E 56, 5594 (1998).

[19] Y. Mao, M.E. Cates, and H.N.W. Lekkerkerker, Physica A 222, 10 (1995).

[20] P.B. Warren, J. Phys. I 4, 237 (1994).

[21] P.G. Bolhuis, A. Stroobants, D. Frenkel, and H.N.W. Lekkerkerker, J. Chem. Phys. 107, 1551 (1997).
[22] H.N.W. Lekkerkerker and A. Stroobants, Nuovo Cimento D 16, 949 (1994).

[23] M.P.B. Bruggen, Ph.D. thesis, Universiteit Utrecht, 1998.

[24] H.N.W. Lekkerkerker, P. Buining, J. Buitenhuis, G.J. Vroege, and A. Stroobants, in Observation, Prediction and Simulation of Phase Transitions in Complex Fluids (Ref. [8]), pp. 53-112.

[25] P.G. Bolhuis and D. Frenkel, J. Chem. Phys. 101, 9869 (1994).

[26] G.A. Vliegenthart, A. van Blaaderen, and H.N.W. Lekkerkerker, Faraday Discuss. 112, 173 (1999).

[27] M. Adams, Z. Dogic, S.L. Keller, and S. Fraden, Nature (London) 393, 349 (1998).

[28] M. Adams and S. Fraden, Biophys. J. 74, 669 (1998).

[29] T. Koda, M. Numajiri, and S. Ikeda, J. Phys. Soc. Jpn. 65, 3551 (1996).

[30] T. Koda and H. Kimura, J. Phys. Soc. Jpn. 63, 984 (1994).

[31] A. Stroobants, Phys. Rev. Lett. 69, 2388 (1992).

[32] R. van Roij and B. Mulder, Phys. Rev. E 54, 6430 (1996).

[33] B. Mulder, Phys. Rev. A 35, 3095 (1987).

[34] R. van Roij, P. Bolhuis, B. Mulder, and D. Frenkel, Phys. Rev. E 52, R1277 (1995).

[35] P. van der Schoot, J. Phys. II 6, 1557 (1996).

[36] X. Wen and R.B. Meyer, Phys. Rev. Lett. 59, 1325 (1987).

[37] T. Koda and S. Ikeda, Mol. Cryst. Liq. Cryst. 318, 101 (1998).

[38] D. Frenkel and B. Smith, Understanding Molecular Simulation (Academic Press, New York, 1996).

[39] T. Koda and S. Ikeda (unpublished).

[40] The smectic order parameter is defined as $\left|\Sigma_{j} e^{i k_{z} r_{j}}\right|^{2} / N^{2}$ evaluated at $k_{z}$ for which the expression attains its maximum value, where $r_{j}$ is the center of mass of the spherocylinder or sphere, and $k_{z}$ is the wave vector along the spherocylinder's long axis [45].

[41] A. Stroobants, H.N.W. Lekkerkerker, and D. Frenkel, Phys. Rev. A 36, 2929 (1987).

[42] P.G. Bolhuis and D. Frenkel, J. Chem. Phys. 106, 666 (1997).

[43] J.M. Polson and D. Frenkel, Phys. Rev. E 56, 6260 (1997).

[44] A. Poniewierski and R. Holyst, Phys. Rev. A 38, 3721 (1988).

[45] J.A.C. Veerman and D. Frenkel, Phys. Rev. A 43, 4334 (1991). 